\title{
Influencia de los Textos de Química en la Enseñanza y Aprendizaje del Concepto de Valencia
}

\author{
Candelaria N. Tejada ${ }^{(1) \star}$, Diofanor Acevedo ${ }^{(2)}$ y Camilo Gattas ${ }^{(3)}$ \\ (1) Universidad de Cartagena, Facultad de Ingeniería, Programa de Ingeniería Química, \\ Avenida el Consulado, Calle 30 No. 48-152. Cartagena, Bolívar-Colombia \\ (e-mail: candelariatejada@yahoo.com) \\ (2) Universidad de Cartagena, Facultad de Ingeniería, Programa de Ingeniería de Alimentos, \\ Avenida el Consulado, Calle 30 No. 48-152. Cartagena, Bolívar-Colombia \\ (3) Institución Educativa Antonio Nariño. Calle 44, No. 2-23. Montería, Córdoba-Colombia.
}

* Autor a quien debe ser dirigida la correspondencia

Recibido Sep. 3, 2014; Aceptado Nov. 14, 2014; Versión final recibida Dic. 12, 2014

\begin{abstract}
Resumen
El objetivo del presente trabajo fue identificar la influencia que ejercen los textos de química en el proceso de enseñanza/aprendizaje de conceptos, particularmente valencia química. Para ello, se analizó la forma como se aborda el concepto en una muestra de textos de química. Así mismo se evaluó dicha influencia mediante la aplicación de un test de conceptos previos a un grupo de 40 estudiantes. Se evidencia que el concepto de valencia en los textos revisados se presenta a los estudiantes en forma incompleta y estática, lo que posibilita la explicación y comprensión de solamente algunas moléculas. Solo se incluye el concepto de valencia primaria en la gran mayoría de los libros excepto los de Chang y Collage y de Petrucci. Estos son los únicos que involucran los aportes realizados por Alfred Werner, respecto a valencia primaria y secundaria. Se concluye que esto puede estar incidiendo en la forma incompleta con la que los estudiantes conciben el concepto de valencia.
\end{abstract}

Palabras clave: aprendizaje significativo, enseñanza de la química, concepto de valencia, textos de química

\section{Influence of Chemistry Textbooks in the Teaching and Learning of the Concept of Valence}

\begin{abstract}
The aim of this study was to identify the influence of chemistry textbooks in the process of teaching/learning of concepts, particularly chemical valence. For this, the form in which the concept is discussed was analyzed in several chemistry textbooks. Also, the influence of the texts was evaluated by applying a survey to a group of 40 students. It was observed that the concept of valence in the texts considered in his study the concept of valence is presented introduces students in incomplete and static form, which allows to explain and comprehend some simple molecules only. The concept of primary valence is not included in most of the books, except those of Chang and Collage and of Petrucci. They are the only ones that include the contributions by Alfred Werner regarding primary and secondary valence. It is concluded that this may be causing the incomplete form in which student understand the concept of valence.
\end{abstract}

Keywords: significant learning, teaching of chemistry, valence concept, chemistry textbooks 


\section{INTRODUCCIÓN}

La química es la ciencia que estudia la estructura de la materia y sus reacciones. Ciencia que resulta de difícil explicación porque con ella se pretenden comprender unos hechos y fenómenos macroscópicos, incursionando unas explicaciones submicroscópicas (Dos Santos-Fernandes y Fernandes-Campos, 2014). Estudios han encontrado que para los estudiantes el mundo de los átomos, moléculas, redes iónicas, etcétera, es el mismo mundo macroscópico de los materiales y de las sustancias pero en diminuto (Valbuena, 2012; Furió y Furió, 2000). En la enseñanza y aprendizaje de la química cualquiera que sea su especialidad, (orgánica, inorgánica, analítica) siempre se han representado problemas, tanto de orden pedagógico como didáctico, no obstante, se observa que diferentes investigadores en el área de la educación han abordado esta temática, desde distintas ópticas, algunos revisan el rol del maestro y sus formas de enseñar, otros indagan sobre las formas como los estudiantes aprenden desarrollando trabajos donde comparan la eficacia del proceso de aprendizaje utilizando herramientas didácticas, tales como: mapas mentales, modelos mentales, mentefactos, revisión de conceptos, de modelos didácticos entre otros, con estudiantes que aprenden en el modelo tradicional el cual se fundamenta principalmente en la repetición y la memoria (Tejada et al., 2013a).

El conocimiento de conceptos en química como la valencia primaria y secundaria es importante para la comprensión de las propiedades físicas y químicas de las sustancias. Conceptos relacionados con este contenido en la química están asociados a fenómenos y procesos diversos. Los problemas de aprendizaje de este tipo de conceptos por parte de los estudiante se revelan en su incapacidad de relacionar estos conceptos con los tres niveles de conocimiento químico: representacional, macroscópico y microscópico (Dos Santos-Fernandes y Fernandes-Campos, 2014; Fernandes et al., 2010). Aunque en la actualidad, los profesores dispongan de metodologías diversificadas para la Enseñanza de la Química como, por ejemplo, las Tecnologías de la Información y Comunicación - TICs, muchas prácticas, incluso hoy, se basan en la transmisión de información, teniendo como recurso exclusivo el libro de didáctico (Proszek y Ferreira, 2009). Pero en la última década, investigadores en docencia de la Química y autores de los textos que los docentes más usan, tales como Chang y Collage (1999), Petrucci (1999), entre otros textos, han dejado fuera de los programas el concepto de valencia secundaria, lo cual refleja la importancia de abordar mejor el concepto de valencia en los cursos de Química preuniversitarios o universitarios.

De otra parte Castillo et al., (2013) afirman para promover el aprendizaje significativo de la Química el docente en la estructura cognitiva del estudiante debe incorporar los aspectos sobre comprensión de los contenidos y la memoria a largo plazo; mientras que lo referente a la funcionalidad de lo aprendido puede vincularse con la actitud afectiva y motivacional. El concepto de valencia, y consecuente explicación de los modelos atómicos, tiene un carácter subjetivo y múltiple, debido a los diferentes enfoques que le han dado los autores de los textos de química, que como fuente primaria de información a través de su contenido inciden en la forma como lo enseñan los profesores, quienes realizan su propia interpretación, que a su vez repercute en la comprensión que haga el estudiante (Tejada et al., 2013b).

\section{MATERIALES Y MÉTODOS}

El enfoque investigativo es de tipo cualitativo desde una perspectiva histórico-cultural debido a que se indagó sobre el desarrollo histórico del concepto de valencia. Se realizó la revisión de una muestra de textos organizados en tres categorías: tradicionales de la enseñanza en el Bachillerato, tradicionales de enseñanza en la formación universitaria y modernos en la enseñanza universitaria. Se seleccionó el concepto de valencia química y se extractaron las definiciones encontradas sobre el concepto de valencia. Esto se complementó con la técnica de test de conceptos previos aplicada a un grupo de 40 estudiantes universitarios de segundo semestre en el curso de química inorgánica; donde se enfrentaron a preguntas abiertas, tales como: Explica, como se forma un enlace químico. Qué diferencias hay entre enlace iónico y covalente. ¿Qué significado tiene para ti el concepto de valencia Química? ¿Conoces la propuesta de Alfred Werner sobre el concepto de Valencia Química? Si tu respuesta es Sí. Explica tu respuesta. Como explicas la existencia de los siguientes moléculas: Cloruro de Sodio $\mathrm{NaCl}$, Ácido Clorhídrico $\mathrm{HCl}$, Hexaamíncobalto (III) $\mathrm{CoCl} 36 \mathrm{NH} 3$, Sulfato de triamínclorodinitroplatino (IV) - [PtCl(NO2)2 (NH3)3](SO4)2; lo anterior teniendo en cuenta que estos conocimientos forman parte del contenido curricular de la asignatura en estudio.

\section{RESULTADOS Y DISCUSION}

La Tabla 1 resume algunos conceptos de valencia presentados en la literatura. Del análisis de los textos revisados se destacan algunos aspectos relevantes. Hay una gran diferencia entre la definición de valencia presentada en los textos tradicionales de la enseñanza en el bachillerato y los textos modernos de la enseñanza universitaria. 
Tabla 1: Revisión de textos de química respecto al concepto de valencia.

\begin{tabular}{|c|c|c|}
\hline Tipo de texto & Nombre del Texto & Definición de Valencia \\
\hline \multirow[t]{2}{*}{$\begin{array}{l}\text { Textos } \\
\text { tradicionales } \\
\text { de la } \\
\text { enseñanza en } \\
\text { el Bachillerato }\end{array}$} & $\begin{array}{l}\text { Química I - Editorial PIME (No } \\
\text { se conoce año de edición ni } \\
\text { autor, dada su antigüedad). }\end{array}$ & $\begin{array}{l}\text { El sustantivo valencia se usa en la química con varios significados; } \\
\text { para evitar confusiones aquí lo usaremos como adjetivo en la } \\
\text { expresión de electrones de valencia. Estos son los electrones más } \\
\text { externos o menos atraídos por el núcleo y que pueden variar en los } \\
\text { átomos al interaccionar. }\end{array}$ \\
\hline & $\begin{array}{l}\text { Química I Grado } 10 \\
\text { (Gutiérrez, 1984). }\end{array}$ & $\begin{array}{l}\text { Valencia es la capacidad de combinación que presentan los átomos. } \\
\text { Los electrones de valencia son os que encontramos en los últimos } \\
\text { niveles de energía. Cuando se hace una combinación, los } \\
\text { electrones de valencia pasan de la capa externa de un átomo a la } \\
\text { del otro o son compartidos por las capas externas de los átomos } \\
\text { que entran en la combinación. }\end{array}$ \\
\hline \multirow{4}{*}{$\begin{array}{l}\text { Textos } \\
\text { tradicionales } \\
\text { de enseñanza } \\
\text { en la } \\
\text { formación } \\
\text { universitaria. }\end{array}$} & $\begin{array}{l}\text { Química general moderna } \\
\text { (Babor e lbarz, 1958). }\end{array}$ & $\begin{array}{l}\text { La valencia de un elemento o capacidad de combinación se expresa } \\
\text { cuantitativamente, por el número de átomos de hidrogeno que se } \\
\text { combinan o son sustituidos por un átomo del elemento. }\end{array}$ \\
\hline & $\begin{array}{l}\text { Química general (Slabaugh y } \\
\text { Parsons, 1972). }\end{array}$ & $\begin{array}{l}\text { Los números de valencia son números enteros chicos para poder } \\
\text { escribir las formulas correctas de muchas sustancias. El número de } \\
\text { coordinación, es el número de vecinos más cercanos unidos por } \\
\text { ligadura combinada al ión metálico central y la valencia del ión } \\
\text { central es la carga positiva formal que adquiere por perdida de } \\
\text { electrones antes de formar un complejo de coordinación. }\end{array}$ \\
\hline & $\begin{array}{l}\text { Fundamentos de Química } \\
\text { general (Garzón, 1982) }\end{array}$ & $\begin{array}{l}\text { El termino valencia se usa para describir el poder que tiene un } \\
\text { átomo para combinarse con otro. El átomo que se emplea como } \\
\text { referencia es el átomo de hidrogeno y por tanto la valencia de un } \\
\text { elemento se define como el número de átomos de hidrogeno que se } \\
\text { pueden combinar con un átomo de ese elemento. }\end{array}$ \\
\hline & $\begin{array}{l}\text { Química general (Briceño, } \\
\text { 1994) }\end{array}$ & $\begin{array}{l}\text { Cada átomo no puede hacer sino determinado número limitado de } \\
\text { enlaces, que es lo que se llama valencia o capacidad de enlace. }\end{array}$ \\
\hline \multirow{5}{*}{$\begin{array}{l}\text { Textos } \\
\text { modernos de } \\
\text { enseñanza } \\
\text { universitaria }\end{array}$} & $\begin{array}{l}\text { Química y ambiente } \\
\text { (Cárdenas y Gálvez, 1999) }\end{array}$ & $\begin{array}{l}\text { Han surgido dos teorías con base en la concepción quántica del } \\
\text { átomo; la del enlace de valencia y la del orbital molecular. }\end{array}$ \\
\hline & $\begin{array}{l}\text { Chang, R. y W. Collage. } \\
\text { Química, décima edición, } 959 \\
\text { p. Editorial McGraw-Hill, } \\
\text { México (2010). }\end{array}$ & $\begin{array}{l}\text { Los químicos del siglo XIX estaban desconcertados por cierto tipo } \\
\text { de reacciones que parecía contravenir la teoría de valencia. } \\
\text { Ejemplo: la valencia del cobalto III y del amoniaco parecían estar } \\
\text { satisfechas, sin embargo al reaccionar forman un compuesto } \\
\mathrm{CoCl}_{3} .6 \mathrm{NH}_{3} \text {. Para explicar esto Alfred Werner explicó que la } \\
\text { mayoría de los elementos tenían dos tipos de valencias. Valencia } \\
\text { primaria y valencia secundaria. El término actual para la valencia } \\
\text { corresponde al número de oxidación y la valencia secundaria } \\
\text { equivale ahora al número de coordinación. Para Werner el cobalto } \\
\text { tiene como valencia primaria } 3 \text { y como valencia secundaria } 6 \text { en el } \\
\text { compuesto } \mathrm{CoCl}_{3} 6 \mathrm{NH}_{3} \text {. }\end{array}$ \\
\hline & $\begin{array}{l}\text { Petrucci, R., Herring, G., } \\
\text { Madura, J., Bissonnette, C. } \\
\text { Química general, décima } \\
\text { edición, } 1071 \text { p. Editorial } \\
\text { Pearson Educación, Madrid } \\
\text { (España) (2011) }\end{array}$ & $\begin{array}{l}\text { La parte principal de la teoría de Werner, propuesta en } 1893 \text {, fue } \\
\text { que algunos átomos metálicos, fundamentalmente metales de } \\
\text { transición, tienen dos tipos de valencia o capacidad de enlace. La } \\
\text { valencia primaria, basada en el número de electrones que el átomo } \\
\text { pierde para formar el ión metálico, y la valencia secundaria que es el } \\
\text { responsable del enlace con otros compuestos, denominados } \\
\text { ligandos, al ión metálico central. }\end{array}$ \\
\hline & $\begin{array}{l}\text { Química para ingeniería } \\
\text { ambiental (Clair y Perry, } \\
\text { 2000). }\end{array}$ & $\begin{array}{l}\text { La valencia o número de oxidación de un átomo está determinada } \\
\text { por el número de electrones que puede tomar, ceder o compartir } \\
\text { con otros átomos... el anillo más externo del átomo contiene el } \\
\text { número de electrones que indica la valencia. }\end{array}$ \\
\hline & $\begin{array}{l}\text { Química conceptos y } \\
\text { problemas (Mosqueira, 2005) }\end{array}$ & $\begin{array}{l}\text { Valencia o número de combinación de un elemento, es el número } \\
\text { de enlaces que un átomo del elemento puede formar con otros } \\
\text { átomos. }\end{array}$ \\
\hline
\end{tabular}


Y aunque en el libro Química I de Grado 10 de Gutiérrez (1984), se utiliza el término "combinación”, al leer la definición, este término no se está usando en el sentido amplio de su significado frente a la definición que hoy, decimos es completa de valencia, sino en el simple para significar unión entre dos átomos compartiendo electrones de su capa más externa (valencia primaria). En este sentido el concepto se presenta acabado al igual que en los otros libros de este mismo grupo y que los textos tradicionales de enseñanza en la formación universitaria, exceptuando la definición presentada por el libro Química General de Slabaugh y Parsons (1972), en el que se presentan dos definiciones. Una de ellas corresponde a una muy buena aproximación del concepto de valencia secundaria, si tenemos en cuenta los criterios que hemos definido como característicos de este concepto. Se resalta la intención del autor, de presentar para esta época, una definición más completa y compleja del concepto valencia química.

El desarrollo histórico de este concepto ha tenido grandes cambios paradigmáticos, ya que este concepto resume los esfuerzos de los químicos del siglo XIX. De acuerdo con diversas fuentes (López y Ospina, 2010; Gallego et al., 2004), los investigadores en didáctica de las ciencias han concluido que la inclusión de la historia de las ciencias en su enseñanza es de una alta importancia, ya que esta puede aportar a solucionar problemas tales como la visión distorsionada y los reduccionismos a los que la ciencia ha sido expuesta (López y Ospina, 2010). En los textos tradicionales de enseñanza en el bachillerato como en los tradicionales en la formación universitaria, además, se presenta el concepto valencia aislado de su desarrollo histórico, como bien lo afirman otros autores en sus estudios (López y Ospina, 2010; Gallego et al., 2004), y con el agravante de que la mayoría de los textos revisados presentan una definición diferente, y en nuestra opinión, confusa; lo que dificulta la comprensión, su aprendizaje y no permite que se aplique en otros aspectos fundamentales de la química, como enlace químico y por ende tampoco se puede explicar la formación de moléculas complejas, tanto orgánicas como inorgánicas.

Con relación a los textos modernos de enseñanza universitaria, encontramos términos científicos, como por ejemplo el de “...orbital molecular...” usados en libro de Química y Ambiente de Cárdenas y Gálvez (1999), que interpretamos como un interés por incluir actualizaciones relacionadas con el concepto. Encontramos también los aportes de Alfred Werner, que sin duda, son un gran avance en la consideración histórica, pero, con el gran inconveniente, que aparece al final de los libros y expuesto de manera tangencial. Esto nos indica que la dimensión histórica no ha adquirido aún, la importancia que, consideramos nosotros, tiene dentro de la enseñanza del concepto, pues conlleva a pensar que, si se alcanza a revisar el tema dentro del plan de estudios para el período académico, se habrá cumplido el objetivo, y por el contrario, si no se alcanza a revisar, esto no tendría importancia, dado que es un tema adicional que traen los libros, tanto que se presenta al final de ellos. Dentro de este grupo de textos, (textos modernos de enseñanza universitaria) nos parece preocupante que hay uno que presenta la definición de valencia como sinónimo de número de oxidación de un átomo, como el caso de Clair y Perry (2000), pues por la revisión de literatura que hemos hecho al respecto y por la experiencia profesional en el campo de la enseñanza de la química, identificamos que estos dos conceptos son distintos, aunque están relacionadas. En este contexto es pertinente citar a Quilez y Sanjosé (1996), quien afirma que los libros de texto como material didáctico, responden implícita o explícitamente a unas concepciones sobre la ciencia y sobre la actividad científica, en los cuales sus autores emplean generalmente versiones simplificadas de ella; esto se puede observar en la forma como los diferentes libros de texto analizados presentan el concepto de valencia en forma acabada, redactado o plasmado en cada uno de forma diferente y muy simplificado.

Se pudo identificar que para referirse a valencia, los textos utilizan palabras tales como: sustantivo, adjetivo, término, concepto, numero, es decir, no encontramos consenso en la terminología utilizada para definir el concepto; sin embargo si encontramos mucha similitud en la idea que transmiten, se centran principalmente en expresar la valencia como la capacidad de combinación o capacidad de enlace (unos hacen referencia a átomos y otros a elementos y esto se presta para confusión). En nuestra opinión, tal parece que este grupo de textos trabajara en cadena el concepto, con lo cual se transmite de manera incompleta y se contribuye en que la enseñanza se haga de igual manera. De otra parte se pudo identificar que en los textos tanto de bachillerato, como de educación superior no hay homogeneidad en la forma como presentan el concepto de valencia, algunos lo relacionan con la capacidad de combinación, otros con el número de enlaces que puede formar un átomo y otros simplemente, expresan que valencia son números enteros chicos, lo cual no le dice mucho al estudiante cuando lee esta información; otros no lo mencionan como tal, sino que hacen referencia a enlaces de valencia o electrones de valencia.

Lo que se cita en el cuadro anterior de Chang y Collage (2010) y Petrucci (2011), parece muy importante porque permite identificar que en la enseñanza del concepto de valencia hay un problema curricular, pues de todos los textos de química revisados, estos dos son los únicos que involucran los aportes realizados por Alfred Werner, respecto a valencia primaria y valencia secundaria, pero esto está muy al final de los textos en un capítulo dedicado a química inorgánica, específicamente química de coordinación; los demás textos ni siquiera incluyen el capítulo de química inorgánica; sin embargo estos mismos textos, abordan el 
concepto tradicional de valencia (capacidad de enlace) en los primeros capítulos donde abordan enlace químico, nomenclatura y tabla periódica, quedando totalmente desarticulado el concepto de valencia propuesto por Alfred Werner lo que está influyendo en que no se perciba el concepto de manera integrado y completo.

Solo se identificó un solo texto, el de Mosqueira y Mosqueira (2005), que le dedica un espacio específico como subtema, y lo desarrolla ampliamente con ejemplos, dentro del capítulo de tabla periódica y enlace químico, sin embargo no integra los aportes de Alfred Werner tan importantes para una comprensión amplia y completa del concepto, a diferencia del resto de los textos que lo abordan como un complemento para explicar otros conceptos, se limitan a un párrafo dentro de un capitulo. Podemos inferir que los textos analizados presentan algunas de las características del concepto identificados anteriormente en la presente investigación, ninguno lo presenta integrando la definición tradicional articulada y complementada con la teoría de Werner y además que este sea presentado en los primeros capítulos del texto, para que sirva de soporte en la comprensión de otros conceptos y para que el estudiante puede entender y explicar la existencia no solo de moléculas sencillas sino también de moléculas complejas, (complejos de coordinación). Pudimos identificar como características abordadas por los textos las siguientes: Capacidad de enlace, número de enlaces, número de oxidación, estas son identificadas por nosotros, más no lo manifiestan explícitamente los textos, lo cual nos parece muy limitado y simplificado para abarcar la explicación del concepto.

Respecto al Test de conceptos previos aplicado a los estudiantes, se pudo establecer que como los textos no están abordando lo referente a los metales de transición, lo cual no se puede explicar satisfactoriamente con el concepto tradicional de valencia, cuando los estudiantes se encuentran con moléculas que involucran metales de transición no tienen soportes conceptuales que les ayude a explicar la existencia de dichas moléculas, esto se pudo evidenciar en el test de conceptos previos cuando se les pregunto a los estudiantes conocimiento básico de los estudiantes sobre el concepto de valencia planteado por Alfred Werner. Respecto a lo anterior se identificó que 39 estudiantes de 40, no conocen la propuesta de Alfred Werner con respecto al concepto de valencia química. Como ejemplo de esto solo un estudiante suministró información sobre la propuesta de Alfred Werner, aunque insuficiente.

Tabla 2: Macrocategorías y subcategorías de las respuesta de estudiantes.

\begin{tabular}{|c|c|}
\hline Macrocategoría & Subcategoría \\
\hline \multirow{4}{*}{$\begin{array}{l}\text { Conceptos básicos sobre } \\
\text { enlace químico y diferencia los } \\
\text { diferentes tipos de enlace }\end{array}$} & Explica la formación de un enlace químico diferenciando entre iónico y covalente. \\
\hline & $\begin{array}{l}\text { Presentan información insuficiente de un enlace químico, así como en la } \\
\text { diferenciación entre estos. }\end{array}$ \\
\hline & $\begin{array}{l}\text { Son imprecisos para explicar la formación del enlace químico, así como en la } \\
\text { diferenciación entre estos }\end{array}$ \\
\hline & No presentan ningún tipo de información \\
\hline \multirow{6}{*}{$\begin{array}{l}\text { Concepciones que tienen los } \\
\text { estudiantes del concepto de } \\
\text { valencia }\end{array}$} & Relacionan la valencia Química con la capacidad de enlace \\
\hline & Relacionan la valencia Química como los electrones del último nivel de energía. \\
\hline & Relacionan la valencia Química con tipo de enlace \\
\hline & Relacionan valencia Química con capa o nivel de energía \\
\hline & Presentan información confusa \\
\hline & No reportan ningún tipo de información. \\
\hline \multirow{2}{*}{$\begin{array}{lll}\text { conocimiento } & \text { básico } & \text { sobre el } \\
\text { concepto } & \text { de } & \text { valencia } \\
\text { planteado por Alfred Werner }\end{array}$} & no conocen la propuesta de Alfred Werner \\
\hline & Suministra información insuficiente sobre la teoría de Alfred Werner, \\
\hline \multirow{6}{*}{$\begin{array}{l}\text { Concepciones de los } \\
\text { estudiantes sobre la formación } \\
\text { de compuestos químicos }\end{array}$} & Explican los compuesto apoyados únicamente en los enlaces iónico y covalente \\
\hline & $\begin{array}{l}\text { Clasifica los compuestos en iónico y covalente, explicando su formación, reconoce } \\
\text { compuestos de coordinación pero no explica su formación }\end{array}$ \\
\hline & $\begin{array}{l}\text { Reconoce los compuestos iónicos y covalentes y explica de manera deficiente los } \\
\text { compuestos de coordinación, haciendo alusión a otras propiedades de los elementos }\end{array}$ \\
\hline & Sin ningún tipo de información \\
\hline & Explica brevemente la formación de enlace iónico \\
\hline & La información Dada no es pertinente \\
\hline
\end{tabular}


De igual forma se evidencia cuando se les preguntó en el test de conceptos previos que explicaran la existencia de algunas moléculas; en este sentido, el grupo no tuvo argumentos para explicar la existencia de moléculas con una mayor complejidad (compuestos de coordinación) Tales como: Hexaamíncobalto (III) CoCl36NH3, Sulfato de triamínclorodinitroplatino (IV) - [PtCl(NO2)2 (NH3)3](SO4)2, pero la gran mayoría si dan razones para explicar moléculas sencillas (tipo $\mathrm{HCl}$ ), todos los argumentos que presentan hacen relación a lo conocido como valencia primaria, la cual permite explicar satisfactoriamente los compuestos sencillos, pero no conocen el concepto de valencia secundaria (propuesta de Alfred Werner) con la cual se pueden explicar la existencia de moléculas complejas como las de los compuesto de coordinación. Las respuestas de los estudiantes se clasificaron en las siguientes categorías: Conocimientos básicos (CB) y errores conceptuales (EC) que tienen los estudiantes, haciéndose necesario incluir los conceptos básicos incompletos $(\mathrm{CBI})$, no responde $(\mathrm{NR})$ denominadas criterios. Las macrocategorías y subcategorías de las respuestas de estudiantes se evidencian en la Tabla 2.

De lo anterior se infiere que los estudiantes creen que el número de electrones determina el número atómico, lo cual no es cierto, pues este último está referido al número de protones presentes en el núcleo del átomo. De otra parte la gran mayoría de los estudiantes asocian varios conceptos a valencia Química, lo que significa que no hay un concepto homogéneo de valencia, atribuyéndole una variedad de concepciones. Así tenemos que para un grupo de estudiantes valencia hace relación al número de electrones del último nivel, para otros, son los niveles de energía, otros la asocian con el tipo de enlace y para otros con la capacidad de enlace. Esto para nuestra investigación es muy significativo, puesto que nos permite hacer aproximaciones sobre el cómo abordaron este conocimiento los docentes además de la didáctica que emplearon para el desarrollo del programa. Se detectó en los estudiantes ausencia de conceptos básicos relacionados con enlace químico.

\section{CONCLUSIONES}

En los textos de química básica general, el concepto de valencia química es manejado de manera tradicional, en forma incompleta y estática, es decir con este concepto solo se posibilita la explicación y comprensión de algunas moléculas (la minoría de ellas) en la que únicamente es posible la interacción de los electrones del ultimo nivel de energía para su formación, y en lo cual participan sólo de elementos de los grupos representativos de la tabla periódica, sin que se permita explicar de esta manera, todos los compuestos que pueden existir en la naturaleza ni tampoco los creados sintéticamente. Como investigadores pensamos que: los textos de química contienen información con corte positivista y contribuyen a la desarticulación curricular del concepto de valencia, los profesores no son conscientes de las limitaciones que se generan en los estudiantes en el proceso de comprensión de la ciencia química, como consecuencia de abordar únicamente el concepto de valencia primaria en los cursos de química básica, porque con ello se limita la construcción de estructuras cognitivas sólidas en química, imposibilitando el aprendizaje significativo. Lo anterior influye en la calidad del aprendizaje de los estudiantes y en términos generales en la comprensión de la ciencia química, puesto que esto no permite que se perciba en forma consciente la verdadera trascendencia que tiene el aprendizaje significativo del concepto de valencia química. Todo esto implica un cambio curricular pues se pudo observar que la mayoría de textos revisados solo presentan al inicio los temas de nomenclatura y enlace químico referidos a ejemplos y casos de moléculas inorgánicas u orgánicas sencillas.

Se propone que cuando se aborde en los textos de química el concepto de valencia se haga en un capitulo exclusivo al inicio del texto y que se tengan en cuenta los siguientes aspectos: (1) Que el concepto de valencia es el producto de la construcción en comunidad, en la que han participado varios científicos, cada uno con su aporte significativo para su época; (2) Que se identifique que La ley de las proporciones múltiples de Dalton es la base para la construcción del concepto de valencia; (3) Que la valencia se considere como la capacidad de combinación de los átomos de los elementos (Postulados de Frankland, quien se refería a Valencia bajo el nombre de atomicidad), siendo este el concepto tradicional de valencia (4) Que se muestre el concepto de valencia como el producto del desarrollo o la evolución de la teoría atómica; (5) La Valencia de un átomo tiene que ver con el número de electrones, presentes en la última capa electrónica; (6) El concepto de enlace químico está relacionado con el concepto de valencia (Valencia primaria y valencia secundaria) y (7) El concepto de valencia secundaria corresponde al número de coordinación y el de primaria al número de oxidación (Alfred Werner).

\section{REFERENCIAS}

Babor J.A. y J. Ibarz, Química general moderna, 6ª ed., 65-66. Editorial Marín, Barcelona, España (1958)

Briceño, C. Química general, 131-133. Editorial Educativa, Bogotá, Colombia (1994) 
Cárdenas, F. y C. Gálvez, Química y ambiente I, Segunda edición, 126-127. McGraw-Hill, Bogotá, Colombia (1999)

Castillo, A., M. Ramírez y M. González, El aprendizaje significativo de la química: condiciones para lograrlo, Omnia, 19(2), 11-24 (2013)

Chang, R. y W. Collage, Química, Séptima edición, 883 p. Editorial McGraw-Hill, México (1999)

Clair, N. y L. Perry, Química para ingeniería ambiental, 14-15. McGraw-Hill, Bogotá, Colombia (2000)

Dos Santos-Fernandes, L. y A. Fernandes-Campos, Enseñanza del enlace químico desde una perspectiva situación-problema, Formación Universitaria, 7(6), 45-52 (2014)

Fernandes, L.S., A.F. Campos y C.A. Marcelino, Concepções alternativas dos estudantes sobre ligação química, Experiências em Ensino de Ciências, 5(3), 19-27 (2010)

Furió, C. y C. Furió, Dificultades conceptuales y epistemológicas en el aprendizaje de los procesos químicos, Educación Química 11(3), 300-308 (2000)

Gallego, R. y otros cuatro autores, El concepto de valencia: su construcción histórica y epistemológica y la importancia de su inclusión en la enseñanza, Ciencia y educación, 10(3), 571-583 (2004).

Garzón, G., Fundamentos de Química general, 103 p. Serie Shaum, Bogotá, Colombia (1982)

Gutiérrez, L., y J. Posada, Química I grado 10, 60p. Educar editores, Bogotá, Colombia (1984)

López, E.C. y J.P. Ospina, El concepto de Valencia: un abordaje histórico y la importancia de la inclusión histórica en la escuela secundaria, MaDoQuim: Memorias de la Maestría en Docencia de la Química, 1(1), 180-187 (2010)

Mosqueira, R.S. y P.S. Mosqueira, Química conceptos y problemas, Segunda edición, 159-160. Editorial Limusa, México (2005)

Pauling, L., The nature of the chemical bond, Journal of Chemical Education, 69(6), 519-521 (1992)

Petrucci, H. Química general, Octava edición, 987p. Editorial Prentice Hall, United States (1999)

Proszek, R. y M. Ferreira, Enseñanza de la Química en Ambientes Virtuales: Blogs, Formación Universitaria, 2(6), 21-30 (2009)

Quilez, J. y V. Sanjosé, El principio de Le Chatelier a través de la historia y su formulación didáctica en la enseñanza del equilibrio químico, Enseñanza de la ciencia, 14(3), 381-390 (1996)

Rios, M. y otros tres autores, Manual de laboratorio de química básica, Segunda edición. Editorial Universidad pontificia Bolivariana., Medellín, Colombia (2005)

Slabaugh. W. y T. Parsons, Química general, 366-367. Editorial Limusa - Wiley, S.A., Mexico (1972)

Tejada, C., C. Chicangana y A. Villabona, Enseñanza de la química basada en la formación por etapas de acciones mentales (caso enseñanza del concepto de valencia), Revista Virtual Universidad Católica del Norte, 38, 143-157 (2013a)

Tejada, C., C. Gattas y A. Villabona, Concepciones alternativas y errores conceptuales de estudiantes sobre modelos atómicos en química, Revista Entornos, 26(2), 267-274 (2013b)

Valbuena, S., Desarrollo de un material didáctico multimedia para facilitar el aprendizaje de química, Revista Educación en Ingeniería, 7(14), 1-9 (2012) 
